\title{
Community minded
}

\author{
Increased local energy generation may offer a route to meet climate targets while empowering community groups, \\ but care is still needed to support those vulnerable to energy system change.
}

\section{ast month, the UK's National Grid}

released its annual Future Energy

Scenarios report ${ }^{1}$, offering its take on possible energy systems for the nation. In a diversion from its normal approach and recognizing recent changes in the energy system, this year National Grid designed their scenarios around the speed and level of decarbonization over the coming decades, rather than prosperity and level of green ambition. Of the four scenarios explored, only two meet the UK's Climate Change Act 2008, which commits the United Kingdom to an $80 \%$ reduction of its greenhouse gas emissions compared to 1990 levels by 2050 . One of these is specifically designed to meet this target; the other is the so-called Community Renewables scenario. There are many similarities between the two, with both relying heavily on smart technology, electric vehicle deployment and a switch to heat pumps, but the degree of energy decentralization is a critical differentiating factor. Larger, centralized generation remains important in the former case, whereas the latter case sees the emergence of a decentralized energy system with up to $65 \%$ of generation occurring at the local level.

This focus on communities and consumers in the Future Energy Scenarios report comes at a time when an increasing amount of attention is being devoted to understanding their role in the energy transition. While the report underscores the potential strengths and benefits of a transition towards greater decentralization, such exercises are not intended to be crystal balls. Indeed, there is a growing body of literature that paints a more complicated picture of the landscape than can be captured in the Community Renewables scenario.

While technological progress in recent years may make community energy seem feasible, social, cultural and policy settings are critical. Social science and humanities research highlights the importance of traditions of social enterprise and stable policy support at a national level as key factors in the success of community energy projects. Engagement in a range of issues, from environmental concerns to social justice and equity, is also crucial to getting projects started and mobilizing individuals.

These factors - and many others under discussion in the literature - highlight an important issue for community energy: it is not the same everywhere. A recent review ${ }^{2}$ reinforces the notion that while there are many societal benefits to community energy, there are barriers to its wider implementation, including specific national circumstances and, perhaps most importantly, a lack of a clear definition of what 'community energy' even means.

At the same time, as much as some communities may come together in the face of increasing technological availability and take matters into their own hands, many communities may not want to engage in this way or are left otherwise vulnerable. The energy transition has its benefits but also its adverse effects, such as job losses and price rises, that mean many miss out, now and in the future. Identifying these communities - regardless of whether or not there is a widespread push towards decentralized energy generation - should be a growing priority for governments.

In their Perspective, Sanya Carley and colleagues at Indiana University, USA offer an example of how this might be done. They adapt the scoping diagram approach, developed to understand vulnerabilities to climate change, to allow them to provide conceptualization of vulnerabilities to the energy transition. This enables them to map out sensitivities of communities to adverse policy effects, such that communities susceptible to adverse energy policy impacts become more apparent, as do those well-placed to adapt. Such mappings may provide an opportunity to identify potential for community energy projects.
Impacts of energy prices also remain an important issue as the energy system evolves. Governments may enact programmes like community energy projects to help empower their citizens, but if people still can't afford to pay their bills or, perhaps worse, are indirectly punished by network cost increases as others switch to decentralized power - then the adverse impacts of the energy transition and its vulnerabilities only deepen.

In a Comment in this issue, Keith Baker at Glasgow Caledonian University and colleagues at Renfrewshire Council and Energy Action Scotland argue that, to be in keeping with an agenda that promotes community empowerment activities like community energy, it is not enough for policies to raise households out of fuel poverty; they must also increase resilience to the very condition of fuel poverty. In this way, they posit the need for more a complex definition of fuel poverty that incorporates dynamic metrics and considers other vulnerabilities in an individual's life that may make them more susceptible to being fuel poor, such as mental or physical health.

If community renewable energy offers a serious opportunity to meet climate targets, as the National Grid's study suggests is the case, then understanding how community energy projects work - and what makes them work - is of utmost importance. But governments should only push for such options if they have a good understanding of who is likely to be affected negatively and how to mitigate that, so they can ensure that vulnerable members of society aren't left behind.

Published online: 8 August 2018 https://doi.org/10.1038/s41560-018-0231-z

\footnotetext{
References

1. Future Energy Scenarios (National Grid, 2018); https://go.nature com/2JWWS2Z

2. Renew. Sust. Energy Rev. 94, 187-196 (2018).
} 